\title{
HIERARCHICAL MARKOV MODELING FOR FUSION OF X RAY RADIOGRAPHIC DATA AND ANATOMICAL DATA IN COMPUTED TOMOGRAPHY
}

\author{
Ali Mohammad-Djafari
}

\author{
Laboratoire des Signaux et Systèmes (CNRS-SUPELEC-UPS) \\ École Supérieure d'Électricité \\ Plateau de Moulon, 91192 Gif-sur-Yvette Cedex, France. \\ E_mail: djafarielss.supelec.fr
}

\begin{abstract}
We consider an $\mathrm{X}$ ray computed tomography (CT) image reconstruction problem where we want to include some geometrical informations coming from anatomical atlas and propose new methods based on hierarchical Markov modeling and Bayesian estimation approach. We use two kinds of anatomical information: partial knowledge of values in some regions and partial knowledge of the edges of some other regions. We show the advantages of using such informations on increasing the quality of reconstructions. We also show some results to analyze the effects of some errors in anatomical data on the reconstructed results.

key words: Data fusion, Markov modeling, Bayesian estimation, Computed tomography, Fusion of radiography and anatomical data.
\end{abstract}

\section{INTRODUCTION}

Data fusion becomes one of the active area of research in medical imaging as well as in many other area such as non destructive testing (NDT), geophysical imaging, process tomography and radioastronomy. We consider here the case of medical imaging with an X ray computed tomography (CT) image reconstruction problem where we want to use two kind of data: classical radiographic (projection) data and some geometrical informations coming from anatomical atlas such as partial knowledge of the anatomic regions and/or the borders of these regions.

The idea of using geometrical data in CT imaging is not new. Many works on the subject has been done before. See for example $[1,2,3,4,5]$. In $[1,5]$, the authors proposed methods for using regions borders from geometrical data in medical imaging and the authors in $[2,3,4]$ used the knowledge of some of regions materials. While the application in the first references concerns medical imaging, the application in the second references concerns industrial NDT. But, combining both regions and borders informations from anatomic data is new.

We give here some preliminary results simulating a fan beam CT problem with more limited number of $X$ ray data and show comparisons of the results using classical back-projection or filtered back-projection methods with those obtained by the proposed method either using or not the anatomical data. These results show the advantages of using anatomical data when these data are exact and well registered with radiographic data. We also show the sensitivity of the proposed method to some errors in anatomical data due to imperfect registration and other uncertainties.

\section{BAYESIAN APPROACH FOR DATA FUSION}

Assume that we are observing an unknown body $\boldsymbol{o}$ through two different measurement systems and obtained two sets of data $\boldsymbol{y}$ and $\boldsymbol{z}$. For example, consider a CT system where $\boldsymbol{y}$ is a set of X ray radiographic data and $\boldsymbol{z}$ a set of ultrasound echo-graphic data. The X ray data are related to the mass density $\boldsymbol{x}$ of the matter while the ultrasound data are related to the acoustic reflectivity $\boldsymbol{r}$ of the matter which is more related to the changes of material mass density inside the object and gives more information on the edges of different homogeneous regions. Indeed, assume that, we have linear relations, both between $\boldsymbol{y}$ and $\boldsymbol{x}$ and between $\boldsymbol{z}$ and $\boldsymbol{r}$. Then we have:

$$
\left\{\begin{array}{l}
\boldsymbol{y}=\boldsymbol{H}_{1} \boldsymbol{x}+\boldsymbol{\epsilon}_{1} \\
\boldsymbol{z}=\boldsymbol{H}_{2} \boldsymbol{r}+\boldsymbol{\epsilon}_{2}
\end{array}\right.
$$

where $\boldsymbol{H}_{1}$ and $\boldsymbol{H}_{2}$ characterize these systems and $\boldsymbol{\epsilon}_{1}$ and $\boldsymbol{\epsilon}_{2}$ their respective modeling and measurement errors.

One approach proposed and used by the author [6] and by other collaborators [4] is based on compound or hierarchical Markovian models where the body object $\boldsymbol{o}$ is assumed to be composed of two related quantities $\boldsymbol{o}=(\boldsymbol{x}, \boldsymbol{r})$, where $\boldsymbol{r}$ is related in some way to $\boldsymbol{x}$, for example $r_{j}=g\left(x_{j+1}-x_{j}\right)$, where $g$ can be any monotonic increasing function.

To account for this relation, we propose to use the following hierarchical model

$$
p(\boldsymbol{o})=p(\boldsymbol{x}, \boldsymbol{r})=p(\boldsymbol{x} \mid \boldsymbol{r}) p(\boldsymbol{r})
$$

which can be used within the Bayes rule to obtain

$p(\boldsymbol{x}, \boldsymbol{r} \mid \boldsymbol{y}, \boldsymbol{z}) \propto p(\boldsymbol{y}, \boldsymbol{z} \mid \boldsymbol{x}, \boldsymbol{r}) p(\boldsymbol{x}, \boldsymbol{r})=p(\boldsymbol{y}, \boldsymbol{z} \mid \boldsymbol{x}, \boldsymbol{r}) p(\boldsymbol{x} \mid \boldsymbol{r}) p(\boldsymbol{r})$

The next step is to choose the prior probability laws $p(\boldsymbol{y}, \boldsymbol{z} \mid \boldsymbol{x}, \boldsymbol{r})$, $p(\boldsymbol{x} \mid \boldsymbol{r})$ and $p(\boldsymbol{r})$. For this, we make the following assumptions:

- Conditional independence of $\boldsymbol{y}$ and $\boldsymbol{z}$ and Gaussian process for $\epsilon_{1}$ and $\epsilon_{2}$ which results to:

$$
p(\boldsymbol{y}, \boldsymbol{z} \mid \boldsymbol{x}, \boldsymbol{r})=p(\boldsymbol{y} \mid \boldsymbol{x}) p(\boldsymbol{z} \mid \boldsymbol{r})
$$

with

$$
\begin{array}{ll}
p\left(\boldsymbol{y} \mid \boldsymbol{x} ; \sigma_{1}^{2}\right) \propto & \exp \left[-\frac{1}{2 \sigma_{1}^{2}}\left\|\boldsymbol{y}-\boldsymbol{H}_{1} \boldsymbol{x}\right\|^{2}\right] \\
p\left(\boldsymbol{z} \mid \boldsymbol{r} ; \sigma_{2}^{2}\right) \propto & \exp \left[-\frac{1}{2 \sigma_{2}^{2}}\left\|\boldsymbol{z}-\boldsymbol{H}_{2} \boldsymbol{r}\right\|^{2}\right]
\end{array}
$$

- Markovian model for $\boldsymbol{x} \mid \boldsymbol{r}: \quad p(\boldsymbol{x} \mid \boldsymbol{r}) \propto \exp [-\alpha \phi(\boldsymbol{x} \mid \boldsymbol{r})]$

- A non Gaussian model for $\boldsymbol{r}: \quad p(\boldsymbol{r}) \propto \exp [-\beta \psi(\boldsymbol{r})]$.

Using these prior laws, we obtain

$$
p(\boldsymbol{x}, \boldsymbol{r} \mid \boldsymbol{y}, \boldsymbol{z}) \propto \exp [-J(\boldsymbol{x}, \boldsymbol{r})]
$$

with

$$
\begin{aligned}
J(\boldsymbol{x}, \boldsymbol{r})= & \frac{1}{2 \sigma_{1}^{2}}\left\|\boldsymbol{y}-\boldsymbol{H}_{1} \boldsymbol{x}\right\|^{2}+\frac{1}{2 \sigma_{2}^{2}}\left\|\boldsymbol{z}-\boldsymbol{H}_{2} \boldsymbol{r}\right\|^{2} \\
& +\alpha \phi(\boldsymbol{x} \mid \boldsymbol{r})+\beta \psi(\boldsymbol{r}) .
\end{aligned}
$$

Many schemes can then be proposed to estimate either $\boldsymbol{x}$ or both $(\boldsymbol{x}, \boldsymbol{r})$. We mention here only the two simplest:

- Simultaneous estimation of both unknowns with the joint MAP estimation (JMAP):

$$
(\widehat{\boldsymbol{x}}, \widehat{\boldsymbol{r}})=\arg \max _{(\boldsymbol{x}, \boldsymbol{r})}\{p(\boldsymbol{x}, \boldsymbol{r} \mid \boldsymbol{y}, \boldsymbol{z})\}
$$


by using an alternate optimization scheme such as:

$$
\left\{\begin{array}{l}
\widehat{\boldsymbol{r}}^{(k)}=\arg \max _{\boldsymbol{r}}\left\{p\left(\widehat{\boldsymbol{x}}^{(k-1)}, \boldsymbol{r} \mid \boldsymbol{y}, \boldsymbol{z}\right)\right\} \\
\widehat{\boldsymbol{x}}^{(k+1)}=\arg \max _{\boldsymbol{x}}\left\{p\left(\boldsymbol{x}, \widehat{\boldsymbol{r}}^{(k)} \mid \boldsymbol{y}, \boldsymbol{z}\right)\right\}
\end{array}\right.
$$

- First estimate $\boldsymbol{r}$ using only $\boldsymbol{z}$ and then use them to estimate $\boldsymbol{x}$ :

$$
\left\{\begin{array}{l}
\widehat{\boldsymbol{r}}=\arg \max _{\boldsymbol{r}}\{p(\boldsymbol{r} \mid \boldsymbol{z})\} \\
\widehat{\boldsymbol{x}}=\arg \max _{\boldsymbol{x}}\{p(\boldsymbol{x} \mid \boldsymbol{y}, \widehat{\boldsymbol{r}})\}
\end{array}\right.
$$

where $\quad p(\boldsymbol{r} \mid \boldsymbol{z}) \propto p(\boldsymbol{z} \mid \boldsymbol{r}) p(\boldsymbol{z}) \quad$ and

$$
p(\boldsymbol{x} \mid \boldsymbol{y}, \boldsymbol{r}) \propto p(\boldsymbol{y} \mid \boldsymbol{x}) p(\boldsymbol{x} \mid \boldsymbol{r}) p(\boldsymbol{r}) .
$$

Other schemes are possible [6].

In previous works, we had considered this last scheme with the following prior laws:

- A generalized Gaussian law for $p(\boldsymbol{r})$ :

$$
p(\boldsymbol{r}) \propto \exp \left[-\alpha \sum_{j}\left|r_{j}\right|^{\beta}\right] \quad \text { with } 1 \leq \beta \leq 2,
$$

to catch the pulse shape nature of $\boldsymbol{r}\left(r_{j}\right.$ is almost always equal to zero in homogeneous regions and can take real value in the borders of these regions). This choice, thanks to long-tailed character of this distribution for the values of $\beta$ near to one, gives the possibility to account for the concentration around zero of the histogram of the values of $r_{j}$ while giving the possibility to have large values. Note that $\beta=2$ gives the Gaussian law.

- A hierarchical Markov model for $p(\boldsymbol{x} \mid \boldsymbol{r})$ :

$$
p(\boldsymbol{x} \mid \boldsymbol{r}) \propto \exp \left[-q\left(r_{j}\right) \phi\left(x_{j}-x_{j-1}\right)\right]
$$

where $\phi$ is a positive potential function and $q\left(r_{j}\right)$ is a decreasing function of $\left|r_{j}\right|$, for example

$$
q_{j}=1-\frac{\left|r_{j}\right|}{\max \left(\left|r_{j}\right|\right)}
$$

to translate the link between the two quantities $\boldsymbol{x}$ and $\boldsymbol{r}$, i.e. when $r_{j}$ is low, it is more probable to be in a homogeneous region and when $r_{j}$ is high, it is more probable to be in a transition region (edges).

Based on these choices, we obtain the following scheme:

$$
\widehat{\boldsymbol{r}}=\arg \max _{\boldsymbol{r}}\{p(\boldsymbol{r} \mid \boldsymbol{z})\}=\arg \min _{\boldsymbol{r}}\left\{J_{1}(\boldsymbol{r} \mid \boldsymbol{z})\right\}
$$

with

$$
J_{1}(\boldsymbol{r} \mid \boldsymbol{z})=\left\|\boldsymbol{z}-\boldsymbol{H}_{2} \boldsymbol{r}\right\|^{2}+\lambda_{1} \sum_{j}\left|r_{j}\right|^{\beta}
$$

and

$$
\widehat{\boldsymbol{x}}=\arg \max _{\boldsymbol{x}}\{p(\boldsymbol{x} \mid \boldsymbol{y}, \widehat{\boldsymbol{q}})\}=\arg \min _{\boldsymbol{x}}\left\{J_{2}(\boldsymbol{x} \mid \boldsymbol{y} ; \widehat{\boldsymbol{q}})\right\}
$$

with

$$
J_{2}(\boldsymbol{x} \mid \boldsymbol{y}, \boldsymbol{q})=\left\|\boldsymbol{y}-\boldsymbol{H}_{1} \boldsymbol{x}\right\|^{2}+\lambda_{2} \sum_{j}\left(1-q_{j}\right) \phi\left(x_{j+1}-x_{j}\right) .
$$

Note that, when the hyperparameters $\lambda_{1}>0$ and $1<\beta<2$ are fixed then $J_{1}$ is a convex function of $\boldsymbol{r}$. Then, its optimization can be done by any gradient based algorithm. This is the same for $J_{2}$ if $\phi$ is chosen to be convex and $\lambda_{2}>0$.

\section{FUSION OF RADIOGRAPHIC AND ANATOMICAL DATA}

The previous approach can easily be extended to the CT image reconstruction where we want to include some geometrical information such as partial knowledge of some of the region values and/or some of the positions of edges or region borders. These informations can be obtained, for example, from anatomical atlas in medical imaging or using other measurement systems such as laser or ultrasound echo-graphic data.
The main idea is that, we must use the information we have in those regions, but we must be careful that these informations are subject to uncertainties. Indeed, in those regions we must preserve the given information, but in the regions we do not have information, still we have to use criterion with capabilities of detecting and preserving the edges.

Using some partial knowledge of some regions borders can be considered as a special case of the method proposed in previous section. Here we propose to add a new term to include some partial knowledge about the values of pixels in some specified regions. Because this information may be partial and there may also be some uncertainty on it, we use again probabilistic approach and model this through a probability law

$$
p(\boldsymbol{x} \mid s) \propto \exp \left[-\sum_{j} \mu_{j} \phi_{2}\left(x_{j}-s_{j}\right)\right]
$$

where $s$ is an image containing the attenuation constant values of some of the regions in the body (not forcibly the same regions for which we know the borders) and $\boldsymbol{\mu}$ an image indicating our degree of confidence about the knowledge of values in those regions ( 0 when no knowledge and 1 when high confidence).

In the following, we assume to have sinogram data $\boldsymbol{y}$ and a binary map $\boldsymbol{q}$ containing the borders of some of the regions in the body, $\boldsymbol{s}$ an image containing the attenuation constant values of some of the regions in the body and $\boldsymbol{\mu}$ an image indicating our degree of confidence about the knowledge of values in those regions.

Using this model and the discussions in previous sections, the Bayesian MAP estimation approach comes up with the following criterion to optimize to find the an image $\widehat{\boldsymbol{x}}$ which will be the result of fusion of these data:

$$
\begin{aligned}
J(\boldsymbol{x} \mid \boldsymbol{y}, \boldsymbol{q}, \boldsymbol{s}, \boldsymbol{\mu})= & \left\|\boldsymbol{y}-\boldsymbol{H}_{1} \boldsymbol{x}\right\|^{2} \\
& +\lambda_{1} \sum_{j}\left(1-q_{j}\right) \phi_{1}\left(x_{j+1}-x_{j}\right) \\
& +\lambda_{2} \sum_{j} \mu_{j} \phi_{2}\left(x_{j}-s_{j}\right)
\end{aligned}
$$

A simple choice for $\phi_{1}$ and $\phi_{2}$ is a quadratic $\phi(u)=u^{2}$ or power form $\phi(u)=|u|^{\beta}$. Other choices are possible, for example, all the potential functional forms with edge preserving properties such as

$$
\phi(u)=\left\{2 \ln (\cosh (u)), \quad 2 \sqrt{1+u^{2}}-2\right\}
$$

which are convex $[7,8]$, or

$$
\phi(u)=\left\{\min \left(u^{2}, 1\right), \quad u^{2} /\left(1+u^{2}\right), \quad \ln \left(1+u^{2}\right)\right\}
$$

which are non convex $[9,10,11]$.

The main advantage of choosing convex potential functions with edge preserving properties is to use half-quadratic optimization algorithms $[12,13]$. In fact, it is now well known that, the joint optimization of a criterion such as $K(\boldsymbol{x}, \boldsymbol{q} \mid \boldsymbol{y})$

$$
\begin{aligned}
K(\boldsymbol{x}, \boldsymbol{q} \mid \boldsymbol{y})= & \left\|\boldsymbol{y}-\boldsymbol{H}_{1} \boldsymbol{x}\right\|^{2} \\
& +\lambda_{1} \sum_{j}\left(1-q_{j}\right)\left(x_{j+1}-x_{j}\right)^{2}+\lambda_{2} \sum_{j} \psi\left(q_{j}\right)
\end{aligned}
$$

with respect to $(\boldsymbol{x}, \boldsymbol{q})$ is equivalent of the optimization of $J(\boldsymbol{x} \mid \boldsymbol{y})$

$$
J(\boldsymbol{x} \mid \boldsymbol{y})=\left\|\boldsymbol{y}-\boldsymbol{H}_{1} \boldsymbol{x}\right\|^{2}+\lambda_{1} \sum_{j} \phi\left(x_{j+1}-x_{j}\right)
$$

followed by a straight forward computation of $\boldsymbol{q}$ from $\boldsymbol{x}$, i.e. $q_{j}=$ $g\left(x_{j+1}-x_{j}\right)$ where the expression of $g$ depends on the expression of $\phi$.

We take advantage of this property to propose an iterative algorithm which starts by optimizing (1) with known values of $\boldsymbol{q}$ to obtain $\widehat{\boldsymbol{x}}$, from which we compute a new estimate $\widehat{\boldsymbol{q}}$ using the mentioned property. Then, we replace those values of it by known values of $\boldsymbol{q}$ and use it again in the next iteration.

The whole reconstruction procedure is then the following:

1. Initialize $\boldsymbol{q}=\boldsymbol{q}^{(0)}$;

2. Compute $\boldsymbol{x}$ by optimizing the criterion (1); 
3. Compute a new value for $\boldsymbol{q}$ from $\boldsymbol{x}$ using the properties of half-quadratic criteria;

4. Replace those a priori known values of $\boldsymbol{q}^{(0)}$ in computed $\boldsymbol{q}$ and return to 2 until convergence.

\section{SIMULATION EXPERIMENTS}

Here, we illustrate the application of the proposed methods for the special case of CT medical imaging. The object is a known numerical phantom in $\mathrm{CT}$ which has been proposed by Shepp and Logan [14]. This is a $(256 \times 256)$ image. The sinogram data is obtained by simulating a fan beam tomography with 64 detectors and 128 angular positions over 0 and 360 degrees for the source. The opening angle for the source is 30.4 degrees. The distance between the source and the center of the object is $600 \mathrm{~mm}$ and the dimensions of the reconstructed image is (400 $\mathrm{mm} \times 400 \mathrm{~mm}$ ).

Fig. 1 shows the original object and the associated sinogram data. Fig. 2 shows the reconstruction results by classical backprojection or filtered back-projection methods used in commercial scanners. As it is seen on this figure, these results are not satisfactory for the data gathering configuration we proposed where we are looking for a high resolution image $(256 \times 256)$ from a sinogram data which has only $(128 \times 64)$ data points $(64$ detectors and 128 source positions uniformly distributed in $0,2 \pi)$. We also give here two other results obtained by optimizing the criterion (3) with $\phi$ a quadratic function once over $\mathbf{R}^{n}$ and the second over $\mathbf{R}_{+}^{n}$. In both cases, we used a simple gradient algorithm, but in the second case, we imposed the positivity constraint at each iteration. These results are significantly better than the classical back-projection methods thanks to regularization terms, but they need more computations (approximately two times more computations than a simple back-projection in each iteration).

Fig. 3 shows the reconstruction results using geometrical data. In this figure, a) and b) show the known region and borders data which are assumed available; c) shows the result when only the region data in a) has been used; d) shows the result when only the borders data in b) has been used; and e) and f) are two results when both region and borders data have been used. These two results have been obtained for two different values of confidence for the regions values (different values of $\lambda_{2}$ which is too low at left but it seems to have good value at right).

Obviously, fusion of more anatomical information results in more accurate results when these data are exact. Unfortunately, in practical applications, we need a first step of registration to bring the geometrical informations in the same frames of $\mathrm{X}$ ray radiographic data. In previous simulations, we assumed that this has been done, before starting the reconstruction.

In the following, we give some results to show the sensitivity of the results on some errors of registration. Here we simulated the cases where the geometrical atlas data are obtained with some errors on the orientation of some of the known regions ( \pm 5 degree).

Fig. 4 shows the results obtained with these errors in the geometrical data with the same conditions which are obtained the results of the Fig. 3. In this figure, a) and b) show the errors in regions and borders.
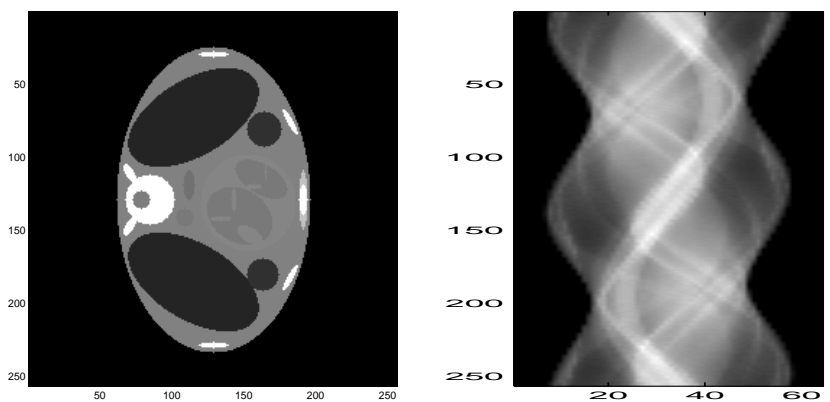

Fig. 1. Original object (left) and its sinogram data (right). The geometry is a fan beam CT, the object is $(256 \times 256)$ and the sinogram data is $(128 \times 64)$.

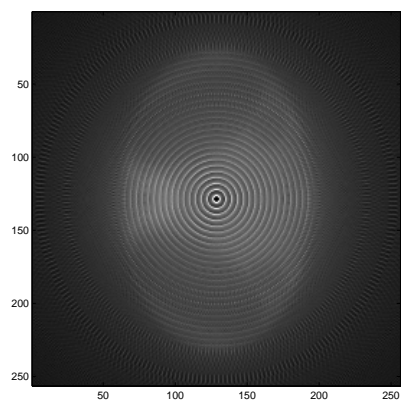

a)

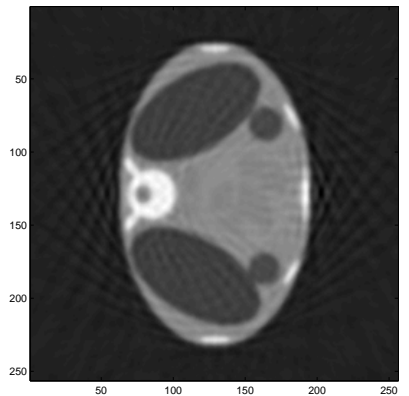

c)

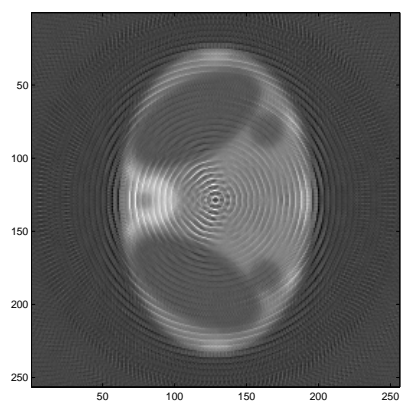

b)

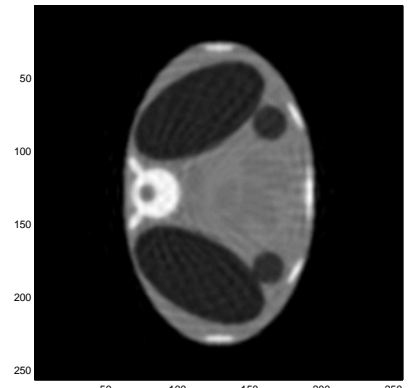

d)
Fig. 2. Reconstructions by classical methods: a) back-projection b) filtered back-projection c) quadratic regularization and d) quadratic regularization with positivity constraint.

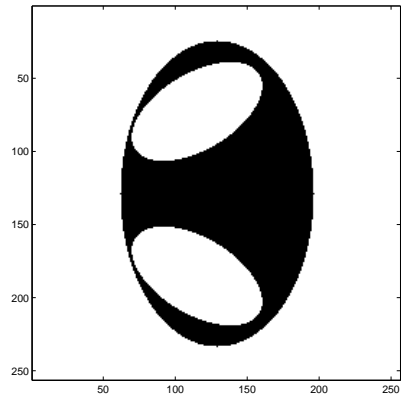

a)

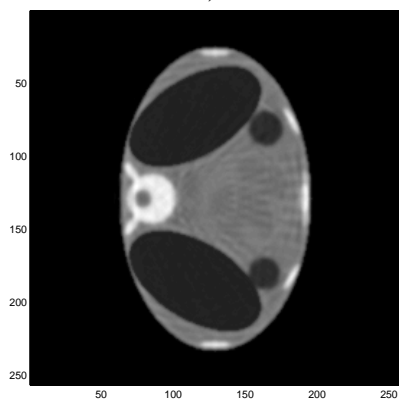

c)

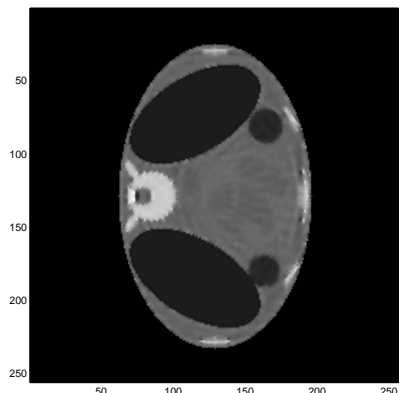

e)

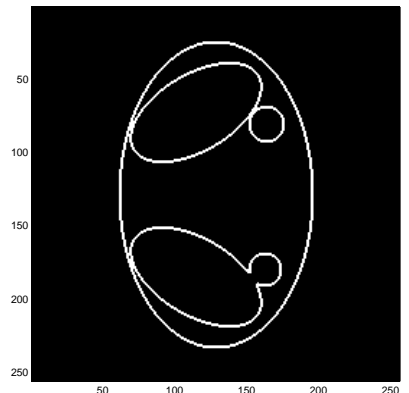

b)

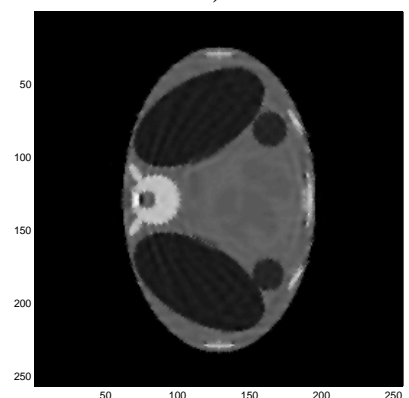

d)

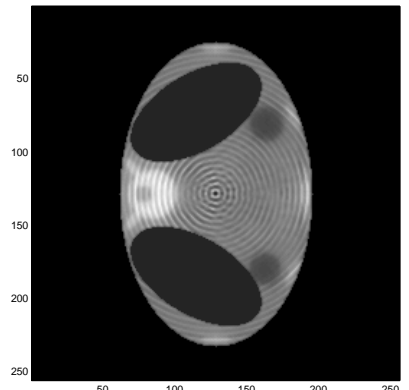

f)
Fig. 3. Reconstructions by data fusion (exact geometrical data): a) known regions data, b) known borders data;

c) Results using a), d) Results using b);

e) and f) Results using both a) and b) for two different values of confidence for the regions values (different values of $\lambda_{2}$ which is too low at left but it seems to have good value at right. 


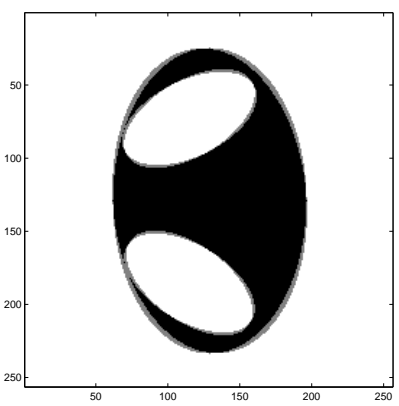

a)

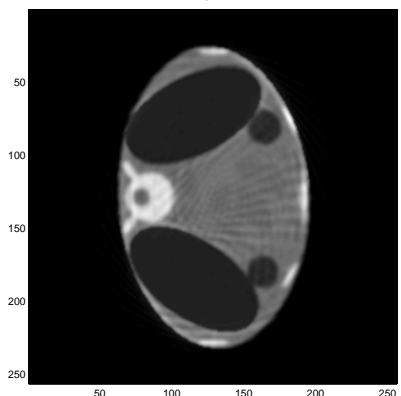

c)

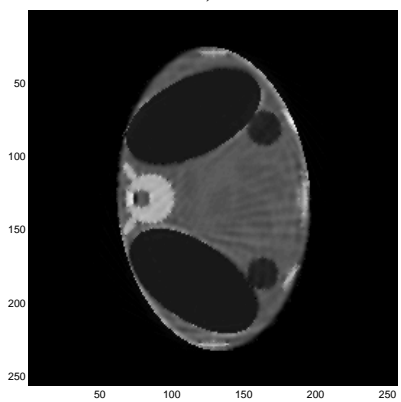

e)

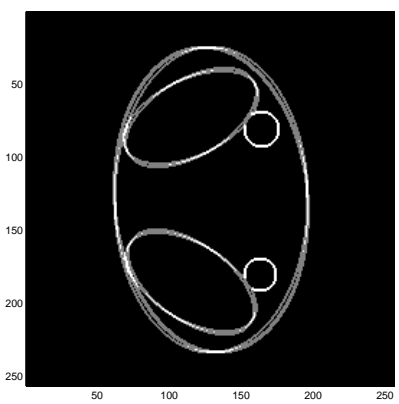

b)

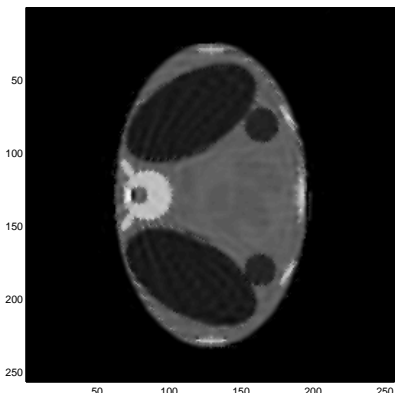

d)

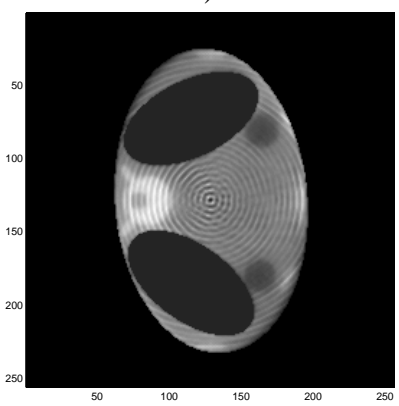

f)
Fig. 4. Reconstructions by data fusion with errors in the geometrical data: a) known regions with errors, b) known borders with errors; c) result using a), d) result using b); e) and f) results using both a) and b) for two different values of confidence for the regions values. These results are to be compared with those of Fig. 3 which were obtained with exact geometrical data.

Comparing these results, we see that the degradations due to these errors are not so crucial if the regularization parameters $\lambda_{1}$ and $\lambda_{2}$ are not too high.

All the previous results have been obtained without feedback procedure for re-estimating the unknown edge positions $\boldsymbol{q}$. Fig. 5 shows a result which is obtained using the iterative procedure explained at the end of the previous section. Comparing the estimated edges on this figure (right) with the a priori known part of these edges in (Fig. 3-b), we see that new region edges have been estimated. These results are still preliminary. Future studies will focus more on the properties of the mentioned iterative algorithm.
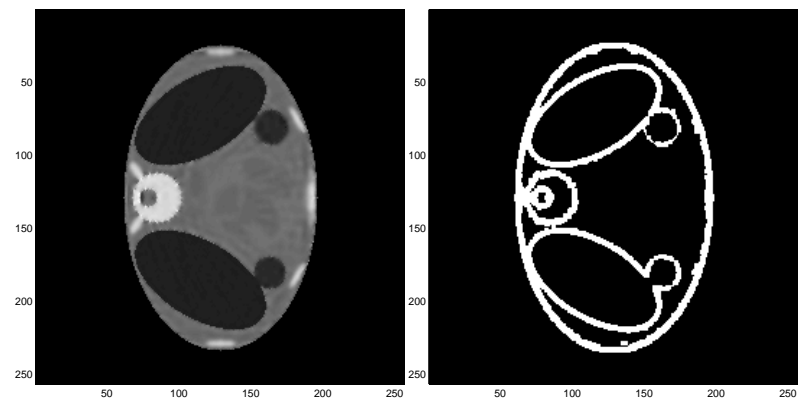

Fig. 5. Reconstructions by incomplete data fusion: This result is obtained using the iterative procedure for re-estimating the edges $\boldsymbol{q}$ : On left $\widehat{\boldsymbol{x}}$ (to be compared with the result on Fig. 3-e) and on right the final re-estimated edges $\widehat{\boldsymbol{q}}$ (to be compared with the known edges on Fig. 3-b).

\section{CONCLUSIONS}

We proposed a hierarchical Markov modeling and a MAP criterion to do data fusion in medical imaging. We illustrated the feasibility of this approach for the case of $\mathrm{X}$ ray computed tomography where we used some anatomical information to obtain better reconstruction results. We used two kind of anatomical information: partial knowledge of values in some regions and partial knowledge of the borders of some other regions. We showed the advantages of using such informations on increasing the quality of reconstructions. We also showed some results to analyze the effects of some errors in anatomic data on the reconstructed results.

\section{REFERENCES}

[1] G. Gindi, M. Lee, A. Rangarajan, and I. G. Zubal, "Bayesian reconstruction of functional images using anatomical information as priors," IEEE Trans. Medical Imaging, vol. MI-12, no. 4, pp. 670-680, 1993.

[2] M. Fiani, J. Idier, and S. Gautier, "Algorithmes ART semiquadratiques pour la reconstruction à partir de radiographies," in Actes du $18^{\mathrm{e}}$ colloque GRETSI, Toulouse, Sept. 2001.

[3] J. Boyd, "Limited-angle computed tomography for sandwich structures using data fusion," Journal of Nondestructive Evaluation, vol. 14, no. 2, pp. 61-76, 1995.

[4] S. Gautier, G. Le Besnerais, A. Mohammad-Djafari, and B. Lavayssière, Data fusion in the field of non destructive testing, Maximum Entropy and Bayesian Methods. Kluwer Academic Publ., Santa Fe, NM, K. Hanson edition, 1995.

[5] S. Som, B. Hutton, and M. Braun, "Edge-preserving regularization based on combined anatomical and functional data," in Joint Workshop: Emerging Computational Methods for Application of SPECT in Cardiology, Neurology and Oncology, Utrecht, Netherland, 1998.

[6] A. Mohammad-Djafari, "Probabilistic methods for data fusion," in Maximum Entropy and Bayesian Methods, Boise, ID, Aug. 1997.

[7] P. J. Green, "Bayesian reconstructions from emission tomography data using a modified EM algorithm," IEEE Trans. Medical Imaging, vol. MI-9, no. 1, pp. 84-93, Mar. 1990.

[8] P. Charbonnier, L. Blanc-Féraud, G. Aubert, and M. Barlaud, "Two deterministic half-quadratic regularization algorithms for computed imaging," in Proc. IEEE ICIP, Austin, TX, USA, Nov. 1994, vol. 2, pp. 168-172.

[9] A. Blake and A. Zisserman, Visual reconstruction, The MIT Press, Cambridge, 1987.

[10] T. Hebert and R. Leahy, "A generalized EM algorithm for 3-D Bayesian reconstruction from Poisson data using Gibbs priors," IEEE Trans. Medical Imaging, vol. 8, no. 2, pp. 194-202, June 1989.

[11] D. Geman and G. Reynolds, "Constrained restoration and the recovery of discontinuities," IEEE Trans. Pattern Anal. Mach. Intell., vol. 14, no. 3, pp. 367-383, Mar. 1992.

[12] D. Geman and C. Yang, "Nonlinear image recovery with half-quadratic regularization," IEEE Trans. Image Processing, vol. 4, no. 7, pp. 932-946, July 1995.

[13] P. Charbonnier, L. Blanc-Féraud, G. Aubert, and M. Barlaud, "Deterministic edge-preserving regularization in computed imaging," IEEE Trans. Image Processing, vol. 6, no. 2, pp. 298-311, Feb. 1997.

[14] B. F. Logan and L. A. Shepp, "Optimal reconstruction of a function from its projections," Duke Math. J., vol. 42, pp. 645-659, 1975. 